\title{
Efficacy of TSPO ligands on neuronal damage mediated by LPS-stimulated BV-2 microglial activation
}

baoyu ma

Weifang Medical University - Fuyanshan Campus: Weifang Medical University

Yongxin Liu

Weifang Medical University

Xiao Zhang

Weifang Medical University

Rui Zhang ( $\square$ zhangrui@wfmc.edu.cn )

Weifang Medical University

Zhenjiang Zhang

Weifang Medical University

Zhihao Zhang

Weifang Medical University

Jie Liu

Weifang Medical University

Zhaodong Juan

Weifang Medical University

Xiaotong Sun

Weifang Medical University

Lina Sun

Weifang Medical University

Jinpeng Feng

Weifang Medical University

Research

Keywords: TSPO, microglia, mitophagy, NLRP3, neuroinflammation, neuron

Posted Date: August 5th, 2021

DOI: https://doi.org/10.21203/rs.3.rs-774268/v1 
License: (c) (i) This work is licensed under a Creative Commons Attribution 4.0 International License. Read Full License 


\section{Abstract \\ Background}

Neuroinflammation mediated by microglia is an important pathological process of neurodegenerative diseases. Alleviating the inflammatory response caused by activated microglia might be a valuable treatment. The $18-\mathrm{kDa}$ translocator protein (TSPO), as a marker of neuroinflammation, is significantly elevated in activated microglia. But the function of TSPO in microglia has not been well demonstrated.

\section{Methods}

In this study, we evaluated the role of TSPO and its ligands in LPS-activated BV-2 microglia involving mitophagy pathway and the nucleotide-binding domain-like receptor protein 3 (NLRP3) inflammasome activation. Then, in the microglia-neuron co-culture system, the neurotoxicity induced by LPS-activated microglia and the neuroprotective effects of TSPO ligands were evaluated.

\section{Results}

Our results showed that after stimulated by LPS, TSPO expression was increased, meanwhile the expression of autophagy associated proteins were decreased in BV-2 microglia cells, but the reduction was reversed by pretreatment with PK11195 and Midazolam. Simultaneously, the NLRP3 inflammasome were increased in LPS activated BV-2 microglia in Transwell co-culture system, pretreatment with TSPO ligands could curb this undesirable situation. Furthermore, TSPO ligands improved the cell viability and reduced apoptosis of neuronal cells in co-culture system.

\section{Conclusions}

TSPO ligands PK11195 and Midazolam showed neuroprotective effects by reducing the inflammatory response of LPS-activated microglia, which may be related to the enhancement of mitophagy and the inhibition of NLRP3 inflammasome.

\section{Background}

The main characteristic of neurodegeneration diseases is chronic progressive loss of neurons in the brain and spinal cord. The pathophysiological processes of neurodegenerative diseases are still unclear. Relevant researches are mainly focused on neuroinflammation ${ }^{1-2}$, abnormal protein conformer ${ }^{3}$, bloodbrain barrier structure and functional integrity disorder ${ }^{4}$. Recent years, the regulation of microglia cell homeostasis about mitophagy and neuroinflammation have become the hotspots in this field ${ }^{5-8}$. 
Microglia are a type of tissue-specific macrophages present in the central nervous system (CNS) and show an immune surveillance function ${ }^{9}$. Plenty of evidence has shown that activated microglia are involved in neuroinflammation ${ }^{10-11}$. As a clinical biomarker of microglial activation and neuroinflammation ${ }^{12}$, TSPO has attracted much attention ${ }^{13-15}$. Analogously, TSPO expression was increased in neurodegenerative diseases ${ }^{16-17}$, in which defective mitophagy is acknowledged as the underlying feature ${ }^{5-8}$, thus suggesting a potential involvement of TSPO in mitochondrial quality control $^{18-19}$ in activated microglia. Decreased efficiency of mitophagy caused by the increase of TSPO ${ }^{19}$ may lead to the accumulation of reactive oxygen species (ROS) which could activate NLRP3 inflammasome to induce inflammatory response ${ }^{20}$. Activated microglia act on the surrounding astrocytes activation to recruit together peripheral macrophages into the brain and amplify the inflammatory response in CNS, the result inevitable damage of neuronal cells. Therefore, the intervention of neuroinflammation is a promising approach for the prevention and treatment of neurodegenerative diseases.

Our previous studies have shown that TSPO ligands have potential therapeutic effects on neuroinflammation ${ }^{21}$. In the current study, we go ahead to investigate the anti-neuroinflammatory effects and possible mechanisms of TSPO ligands in LPS-activated BV-2 microglial cells in vitro. In that way, the role of mitophagy was looked into. Finally, the protective effects of TSPO ligands against neuroinflammation-induced neurotoxicity in NSC34 neuronal cells and HT-22 neuronal cells both cocultured with BV-2 microglia were explored.

\section{Methods}

Cell Culture and Treatment

Full details on cell lines are provided in the Supplementary Table 1. BV-2 microglia cells were cultured in 1,640 medium, NSC34 and HT-22 cells were cultured in DMEM medium, with $10 \%$ fetal bovine serum (Gibco, Shanghai, China). The BV-2 microglia cells were randomly divided into six groups including Control group, PK11195 (0.5 $\mu \mathrm{M}$, Sigma, Aldrich, USA) group, LPS $(1 \mu \mathrm{g} / \mathrm{mL}$, Solarbio, Beijing, China) group, PK11195 + LPS group, Midazolam (15 $\mu$ M, NHWA, Jiangsu, China) group, Midazolam + LPS group. In both PK11195 + LPS group and Midazolam + LPS group, cells were pretreated with PK11195(0.5 $\mu \mathrm{M})$ or Midazolam $(15 \mu \mathrm{M})$ for $1 \mathrm{~h}$ before treated with LPS for $6 \mathrm{~h}$, and all the cells were harvested after treatment.

BV-2 Microglia and NSC34 Neuronal Cells Co-culture System

NSC34 neuronal cells were co-cultured with conditioned medium (CM) from BV-2 microglia cells or were co-cultured with BV-2 microglia cells in Transwell (24 mm Transwell with $0.4 \mu \mathrm{m}$ pore size insert; Corning, New York, USA) system. For CM co-culture system (Supplementary Fig. 1a), the cells were randomly divided into four groups: Control, LPS, PK11195 + LPS and Midazolam + LPS. In LPS group, BV-2 microglia were activated by $10 \mathrm{ng} / \mathrm{mL}$ LPS. The last two groups, BV-2 microglia cells were pretreated 
with PK11195(0.5 $\mu \mathrm{M})$ or Midazolam $(15 \mu \mathrm{M})$ for $1 \mathrm{~h}$ followed by LPS treatment for $6 \mathrm{~h}$, then, the BV-2 microglia cells were replaced with serum-free culture medium for another $12 \mathrm{~h}$. The microglia culture supernatant continued culture NSC34 neuronal cells for $12 \mathrm{~h}$. For Transwell co-culture system (Supplementary Fig. 1b), the upper insert chamber was planted with BV-2 microglia cells and the lower six-well plate was used to implant NSC34 neuronal cells. In LPS group, BV-2 microglia cells treated with LPS at a concentration of $10 \mathrm{ng} / \mathrm{mL}$ for $6 \mathrm{~h}$. The last two groups, BV-2 microglia cells pretreated with PK11195 $(0.5 \mu \mathrm{M})$ or Midazolam $(15 \mu \mathrm{M})$ for $1 \mathrm{~h}$ followed by LPS treatment for $6 \mathrm{~h}$. Then, two kinds of cells were replaced with serum-free culture medium at the same time. The insert chamber was placed in combination with the six-well plate co-cultivation for $12 \mathrm{~h}$.

BV-2 Microglia and HT-22 Neuronal Cells Co-culture System

As mentioned above, the steps of the BV-2-HT-22 co-culture system (Supplementary Fig. 2) were consistent with those of BV-2-NSC34. The concentration of LPS was $1 \mathrm{ug} / \mathrm{mL}$, the co-culture time was 24 in BV-2-HT-22 co-culture system.

Cell Viability Assay

Cell viability was measured by the Cell Counting Kit-8 (CCK-8) assay. Briefly, cells were seeded in 96-well culture plates and received the various treatments. After that, cells were incubated with $10 \mu \mathrm{L}$ CCK-8 reagent and finally the absorbance at $450 \mathrm{~nm}$ was measured using a microplate reader (BIO-RAD iMark).

Western blot Analysis

Each sample contained with $20 \mu \mathrm{g}$ of protein was separated by $10 \%$ or $12 \%$ SDS-PAGE gels and transferred to PVDF membrane. The PVDF membranes were incubated with the primary antibodies at $4^{\circ} \mathrm{C}$ respectively overnight. Full details on primary antibodies used are provided in Supplementary Table 2. The horseradish peroxidase (HRP)-conjugated secondary antibodies (Goat anti-Rabbit, 1:7000 or Goat anti-Mouse, 1:7000; Proteintech, Wuhan, China) were used for $2 \mathrm{~h}$ at room temperature and protein expressions were evaluated by using the enhanced chemiluminescence plus detection system (Tanon 4600, Shanghai, China).

RNA Isolation and Quantitative Real-Time PCR Assays

Total RNA was extracted with TRIzol reagent (Thermo Fisher Scientific, Shanghai, China) following the

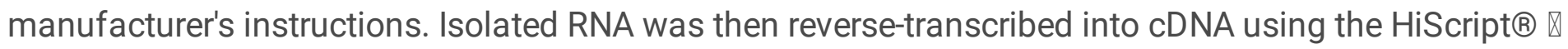
RT SuperMix (Vazyme, Nanjing, China) following the standard protocol. For real-time quantitative PCR analysis, the resultant cDNA products were amplified using a $2 \times$ ChamQ SYBR qPCR Master Mix (Vazyme, Nanjing, China) in triplicate. The mRNA level was normalized to $\beta$-actin and expressed as foldincrease. The forward and reverse primer sequences are shown in Supplementary Table 3.

Immunofluorescence 
After treatment, the cells were treated with $4 \%$ paraformaldehyde for $30 \mathrm{~min}$, then blocked in $5 \%$ goat blocking serum (Solarbio, Beijing, China) for $30 \mathrm{~min}$ at room temperature. Primary antibodies were incubated at $4{ }^{\circ} \mathrm{C}$ overnight. Full details on primary antibodies used are provided in Supplementary Table 4. Then, the cells were incubated with goat anti-rabbit IgG (1:500; Multi Sciences, Hangzhou, China) secondary antibody for $2 \mathrm{~h}$ in the dark at $37^{\circ} \mathrm{C}$. Finally, fluorescence images were captured with a fluorescence microscope (Olympus, Tokyo, Japan), and the analysis of the fluorescence images was performed by Image $\mathrm{J}$.

Transmission electron microscopy (TEM)

The cell precipitation was fixed in ice cold $3 \%$ glutaraldehyde in phosphate buffer, $\mathrm{pH} 7.4$ for $1 \mathrm{~h}$, then post fixed in $1 \%$ OsO4. Following dehydration and embedding in Epon resin, ultrathin sections were cut and stained with uranyl acetate and lead citrate. Finally, the sample were imaged under the transmission electrons microscope (HT 7700-SS, HITACHI, Japan).

Detection of Mitochondrial-Derived DCFH-ROS Levels

After treatment, the BV-2 microglia cells incubated with $10 \mu \mathrm{M}$ DCFH-DA (Beyotime, Shanghai, China) in the dark at $37^{\circ} \mathrm{C}$ for $40 \mathrm{~min}$. After washing the cells for three times with serum-free culture medium, images were captured using a fluorescence microscope (Olympus, Tokyo, Japan) and analyzed with Image J.

Enzyme-Linked Immunosorbent Assay (ELISA)

IL-1 $\beta$ (Multi Sciences, Hangzhou, Zhejiang, China) and IL-18 (Multi Sciences, Hangzhou, Zhejiang, China) that the cells had secreted into the culture supernatant in lower chamber in Transwell co-culture system were measured by ELISA according to the manufacturer's instructions.

\section{TUNEL}

The apoptosis of neuronal cells was detected by CoraLite®594 TUNEL Assay Apoptosis Detection kit (Proteintech Group, Inc) according to the manufacturer's directions. The apoptotic cells were TUNEL positive which were labeled with red fluorescence using a fluorescence microscopy. The ratio of TUNELpositive cells to total neuronal cells indicates the apoptosis index.

Statistical analyses

Data are presented as means \pm SEM of three independent experiments. The data were analyzed with oneway ANOVA followed by Tukey's post hoc test for significance via SPSS 25.0. Asterisks indicate statistically significant differences between the compared groups: $P<0.05$.

\section{Results}


TSPO Ligands Attenuated LPS-induced high TSPO expression in BV-2 Microglial cells

We first investigated whether treatment with the LPS $(1 \mu \mathrm{g} / \mathrm{mL})$ mediated the TSPO production in BV-2 microglia cells. As shown in Fig. 1. (a-d), the Western blot, immunofluorescence and qRT-PCR results show that compared with Control group, TSPO protein and mRNA level significantly enhanced in LPS group, but not significantly affected in PK11195 and Midazolam group. Interestingly, compared with the LPS group, both the TSPO expression in the PK11195 + LPS group and the Midazolam + LPS group were reduced. Next, we tested the expression of TSPO in microglia in the BV-2-NSC34 Transwell co-culture system. As shown in Fig. 1. (e-h), we found that LPS $(10 \mathrm{ng} / \mathrm{mL})$ stimulation caused TSPO overexpression in BV-2 microglia of the co-culture system, PK11195 or Midazolam pretreatment could alleviate the increase of TSPO.

TSPO Ligands Reversed the Inhibition of mitophagy in LPS-Activated BV-2 Microglial Cells

To figure out the role of mitophagy in the regulation of microglia activation and neuroinflammation, we assessed the effects of LPS on the autophagic process. The Western blot and qRT-PCR results showed that exposed to LPS $(1 \mu \mathrm{g} / \mathrm{mL})$ could impaired mitophagy in BV-2 microglia. Compared with Control group, the expression of autophagy-related protein ATG7(Fig. 2a, c) and ATG5(Fig. 2a, e) protein decreased; the expression of p62(Fig. 2b, f) increased in LPS group. Immunofluorescence results also showed that compared with Control group, the ATG7(Fig. 2g) and LC3B (Fig. 2h) protein intensity was significantly attenuated, the p62(Fig. 2i) protein intensity was increased in the LPS group. However, PK11195 or Midazolam pretreatment significantly reversed the inhibition of mitophagy by LPS. qRT-PCR results are similar as Western blot results, ATG7(Fig. 2j) mRNA and LC3B (Fig. 2k) mRNA expression were decreased; the expression of p62(Fig. 2l) mRNA increased. Further, TEM results (Fig. $2 \mathrm{~m}$ ) showed autophagic vacuoles containing swollen mitochondria were present in LPS-activated BV-2 microglia cells (white solid arrow). While cells pretreated with PK11195 or Midazolam followed by exposure of LPS, more mitochondria with vacuolation and more autophagic vacuoles (black solid arrow) were observed.

TSPO Ligands Inhibited Neuroinflammatory Reactions in LPS-Activated BV-2 Microglial Cells in Transwell co-culture system

Our previous research pointed out that TSPO ligands can inhibit the activation of NLRP3 inflammasome in LPS activated BV-2 microglia. Next, our present study further detected the activation of NLRP3 inflammasome in LPS activated BV-2 microglia in BV-2-NSC34 Transwell co-culture system. The results (Fig. 3a) showed that the levels of ROS were significantly increased in BV-2 microglia cells. PK11195 or Midazolam pretreatment significantly inhibited the expression of ROS stimulated by LPS. Further, the levels of NLRP3, Cleaved caspase-1 were measured by Western blot, qRT-PCR, and immunofluorescence. The results (Fig. 3b-g) showed that PK11195 or Midazolam pretreatment significantly inhibited the protein expression of NLRP3, Cleaved caspase-1 and the mRNA expression of NLRP3 in BV-2 microglia. Moreover, the results (Fig. 3h-i) showed that the mRNA levels of IL-1 $\beta$ and IL-18 were significantly increased, pretreatment with PK11195 or Midazolam effectively inhibited the mRNA expression. Then, in BV-2-NSC34 Transwell co-culture system (Fig. 3j-k), we found that the pretreatment could significantly 
reduce the content of IL-1 $\beta$ and IL-18 in the supernatant. Likewise, the pretreatment could significantly reduce the content of IL-1 $\beta$ and IL-18 in lower chamber in BV-2-HT-22 Transwell co-culture system.

TSPO ligands Prevented LPS-Activated Microglia Induced Neurotoxicity in BV-2-NSC34 co-culture system

We then investigated whether TSPO ligands could prevent neuroinflammation mediated neuronal damage using the BV-2 microglia CM and the BV-2-NSC34 co-culture system. First, we use different concentrations of LPS to stimulate microglia to produce CM to process NSC34 neuronal cells, so as to select the appropriate LPS stimulation concentration. As shown in the Fig. $4 \mathrm{a}$, we selected $10 \mathrm{ng} / \mathrm{mL}$ LPS stimulated microglia in the BV-2 CM or BV-2-NSC34 Transwell co-culture system. Expectedly, illustrated in Fig. 4b pretreatment with PK11195 and Midazolam prevented NSC34 neuronal cells against activated microglia-induced cytotoxicity, increased the neuronal cell viability. Further, we used Western blot to detect the expression of apoptosis-related proteins Bcl-2(B-cell lymphoma-2), Bax (Bcl2-Associated X), Cleaved caspase-3 in NSC34 neuronal cells. The results of Bcl-2/Bax indicated that PK11195 and Midazolam pretreatment could protect against NSC34 neuronal cell apoptosis, as evidenced by increasing in ratio of $\mathrm{Bcl}-2 / \mathrm{Bax}$ compared with the LPS-treated group in CM (Fig. 4c) or Transwell (Fig. 4e) co-culture system. In addition, compared with control group, LPS stimulation significantly increased Cleaved caspase-3 activity in NSC34 neuronal cells in CM (Fig. 4d) and Transwell co-culture system (Fig. 4f), whereas PK11195 and Midazolam incubation resulted in reduction of Cleaved caspase-3 expression. Meanwhile, TUNEL staining was used to monitor the apoptosis of NSC34 neuronal cells, LPS stimulation induced the increase of apoptosis positive cell. These changes were attenuated by pretreatment with PK11195 and

Midazolam evidenced by decreased number of apoptosis positive cells in CM (Fig. $4 \mathrm{~g}$ ) or Transwell (Fig. 4h) co-culture system.

TSPO ligands Prevented LPS-Activated Microglia Mediated Neurotoxicity in BV-2-HT-22 co-culture system

Likewise, as shown in Fig. 5a, we selected $1 \mu \mathrm{g} / \mathrm{mL}$ LPS stimulated microglia in the BV-2 CM or BV-2-HT22 Transwell co-culture system. Illustrated in Fig. 5b, pretreatment with PK11195 and Midazolam prevented HT-22 neuronal cells against activated microglia-induced cytotoxicity in CM co-culture system. Then, found that PK11195 and Midazolam pretreatment could increase the ratio of Bcl-2/Bax compared with the LPS group in CM (Fig. 5c) or Transwell (Fig. 5e) co-culture system. In addition, PK11195 and Midazolam incubation resulted in reduction of Cleaved caspase-3 expression whether in CM (Fig. $5 \mathrm{~d}$ ) or Transwell (Fig. 5f) co-culture system. As illustrated in Fig. 5g-h, TUNEL staining shown that LPS stimulation induced the increase of apoptosis positive cell. However, these changes were attenuated by pretreatment with PK11195 and Midazolam evidenced by decreased number of apoptosis positive cells.

\section{Discussion}

Our current results showed TSPO ligands PK11195 and Midazolam played an anti-inflammatory role by improving mitophagy to inhibit NLRP3 inflammasome activation in vitro. Meanwhile, in the microglianeurons co-culture system in vitro, TSPO ligands pretreatment can reduce the damage of microglia inflammatory response to neurons, thus playing a neuroprotective role. 
Under normal circumstances, TSPO has low expression in the CNS and high level of TPSO in the brain especially in activated microglia suggests a significant injury and inflammation ${ }^{13,22}$. In vivo human imaging studies have shown that TSPO levels in activated glial cells are increased, especially in neurodegenerative diseases ${ }^{17}$. In present study, BV-2 microglia cells were used for our research. After activation of BV-2 microglia cells by LPS, the expression of TSPO was significantly increased (Fig. 1a-d). Meanwhile, in BV-2-NSC34 Transwell co-culture system, stimulation with LPS also caused high TSPO expression in microglia (Fig. 1e-h). Researches over the last few decades have had a strong emphasis on the interaction of TSPO with its ligands, the most common of which is PK11195 ${ }^{12}$.TSPO ligands have shown promising therapeutic prospects in various studies ${ }^{14-15}$. Previous studied in our laboratory showed PK11195 and Midazolam have anti-inflammatory action in vitro ${ }^{21}$. Meanwhile, in vivo we found that PK11195 could regulate the process of mitophagy ${ }^{23}$. So, we further examined the regulatory effect of PK11195 and Midazolam on mitophagy in microglia in vitro. Mitophagy plays an important role in the development and maintenance of the $\mathrm{CNS}^{23-25}$, which can help cells adapt to the living environment ${ }^{26}$. In current study, Western blot, qRT-PCR, immunofluorescence results (Fig. 2a-I) showed that the expression of mitophagy related proteins ATG7, ATG5, p62, LC3B in LPS activated BV-2 microglia cells were disorganized. Further, images taken with TEM showed (Fig. $2 \mathrm{~m}$ ) that LPS-induced autophagy appeared to occur in the early stages of autophagy formation, since most autophagic vacuoles were observed. In contrast, after pretreatment with PK11195 or Midazolam before exposure to LPS, more mature autophagosomes and advanced autophagic lysosome structures were observed instead, indicating that the damaged mitochondria are further removed and recycled. The dysregulation of the mitophagy pathway could lead to the accumulation of damaged mitochondria, resulting in increased oxidative stress ${ }^{20}$. We found the high levels of ROS in LPS activated BV-2 microglia in BV-2-NSC34 Transwell system. ROS could activate NLRP3 inflammasomes. It has been reported that the expression level of NLRP3 in the brain of elderly rats is significantly higher than that of young rats, and the activation level of NLRP3 is significantly increased in neurodegenerative diseases ${ }^{27-28}$. In our study (Fig. 3), PK11195 and Midazolam pretreatment can inhibit the activation of NLRP3 by reducing the production of ROS. The activation of NLRP3 inflammasome could induce secretion of inflammatory cytokines, such as IL-18, IL$1 \beta^{29}$. So, we investigated the content of IL-18 and IL-1 $\beta$ in Transwell co-culture system and found that the increased content of IL-1 $\beta$ and IL-18 in LPS group (Fig. 3). PK11195 as well as Midazolam could reduce the content of IL-1 $\beta$ and IL-18 secreted by LPS activated microglia.

The release of inflammatory cytokines causes neuronal damage and amplify the inflammatory response in $\mathrm{CNS}^{30}$. And then, we used two types of co-culture systems according to the ideas of our predecessors ${ }^{31-34}, \mathrm{CM}$ co-culture system and Transwell co-culture system, to verify the neuroprotective effects of PK11195 and Midazolam. More than that, two different neuronal cell lines were used to simulate the motor neurons and the hippocampal neurons in this study. Among them, NSC34 cell lines were often used to the study of amyotrophic lateral sclerosis ${ }^{35}$ and HT-22 hippocampal neuron cell lines were often used to study of cognitive impairment ${ }^{32}$ in vitro. The data showed that PK11195 and Midazolam preconditioning alleviated the decrease in neuronal activity induced by LPS-activated 
microglia, including NSC34 and HT-22 neuronal cells in CM co-culture system. At the same time, we detected the apoptosis of NSC34 and HT-22 neuronal cells under two co-culture systems. Our data indicated that PK11195 and Midazolam pretreatment could reduce the apoptosis of NSC34(Fig. 4) and HT-22(Fig. 5) neuronal cells.

Taken together, our current study demonstrated several principal findings, as follows: (1) TSPO expression was increased in LPS activated BV-2 microglia cell, TSPO ligands PK11195 and Midazolam could regulate it expression. (2) TSPO ligands PK11195 and Midazolam conferred anti-inflammatory via improving the quality control of mitochondria and inactivation of NLRP3 inflammasome. (3) TSPO ligands PK11195 and Midazolam showed neuroprotective effects by regulating the microglia cell homeostasis in microglia-neurons co-culture system.

\section{Conclusion}

TSPO ligands PK11195 and Midazolam showed neuroprotective effects by reducing the inflammatory response of LPS-activated microglia, which may be related to the enhancement of mitophagy and the inhibition of NLRP3 inflammasome.

\section{Abbreviations}

TSPO: 18-kDa translocator protein; LPS: Lipopolysaccharide; BV-2: Mouse microglia cells; NLRP3: Nucleotide-binding domain-like receptor protein 3; CNS: Central nervous system; ROS: Reactive oxygen species; NSC34: Mouse motor neuron; HT-22: Mouse hippocampal neuron; CM: Conditioned medium; CCK-8:Cell counting kit-8; TEM: Transmission electron microscopy; DMEM: Dulbecco's modified Eagle's medium; FBS: Fetal bovine serum; ELISA: Enzyme-linked immunosorbent assay; IL-1 $\beta$ : Interleukin-1 $\beta$;IL18: Interleukin-18; ATG7: Autophagy-related protein 7; ATG5: Autophagy-related protein 5; p62:SQSTM1; LC3B: Microtubule-associated protein 1 light chain 3;Bcl-2: B-cell lymphoma-2; Bax: Bcl2-Associated X.

\section{Declarations}

\section{Acknowledgements}

Thanks to school of Anesthesiology, Shandong Provincial Medicine and Health Key Laboratory of Clinical Anesthesia, Weifang Medical University.

\section{Funding}

This work was supported by Natural Science Foundation of Shandong Province (ZR2017MH066, ZR2020MH017, ZR2019PH037) and Weifang medical university research startup fund for $\mathrm{PhD}(2017 \mathrm{BSQD} 28)$.

\section{Availability of data and materials}


The data, analytic methods, and study materials will be made available to other researchers for the purpose of reproducing the results or replicating the procedures. The data that support the findings of this study are available from the corresponding author upon reasonable request. The authors will be responsible for maintaining availability.

\section{Authors' contributions}

Study conception/design: RZ, ZJZ, BM, XZ, YL

Conduct of experiments: BM, XZ, YL, ZHZ, JL, JF

Data analysis: $B M, X Z, Y L, Z H Z, J L, J F$

Drafting of paper: BM, YL

Editing/revision of paper: RZ, ZJZ, ZJ, XS, LS

All authors contributed to the article and approved the submitted version.

\section{Ethics approval and consent to participate}

Not applicable.

\section{Consent for publication}

Not applicable.

\section{Competing interests}

The authors declare no conflicts of interest.

\section{References}

1. Safavynia SA, Goldstein PA. The Role of Neuroinflammation in Postoperative Cognitive Dysfunction: Moving From Hypothesis to Treatment. Front Psychiatry. 2018;9:752. doi:10.3389/fpsyt.2018.00752.

2. Quinn PMJ, Moreira PI, Ambrósio AF, Alves $\mathrm{CH}$. PINK1/PARKIN signalling in neurodegeneration and neuroinflammation. Acta Neuropathol Commun. 2020;8(1):189. doi:10.1186/s40478-020-01062-w.

3. Dugger BN, Dickson DW. (2017). Pathology of Neurodegenerative Diseases. Cold Spring Harb Perspect Biol, 9(7). doi:10.1101/cshperspect.a028035.

4. Sweeney MD, Sagare AP, Zlokovic BV. Blood-brain barrier breakdown in Alzheimer disease and other neurodegenerative disorders. Nat Rev Neurol. 2018;14(3):133-50. doi:10.1038/nrneurol.2017.188.

5. Agrawal I, Jha S. Mitochondrial Dysfunction and Alzheimer's Disease: Role of Microglia. Front Aging Neurosci. 2020;12:252. doi:10.3389/fnagi.2020.00252. 
6. Guo F, Liu X, Cai H, Le W. Autophagy in neurodegenerative diseases: pathogenesis and therapy. Brain Pathol. 2018;28(1):3-13. doi:10.1111/bpa.12545.

7. Niranjan R. Recent advances in the mechanisms of neuroinflammation and their roles in neurodegeneration. Neurochem Int. 2018;120:13-20. doi:10.1016/j.neuint.2018.07.003.

8. Wang D, Zhang J, Jiang W, Cao Z, Zhao F, Cai T,.. . Luo W. The role of NLRP3-CASP1 in inflammasome-mediated neuroinflammation and autophagy dysfunction in manganese-induced, hippocampal-dependent impairment of learning and memory ability. Autophagy. 2017;13(5):914-27. doi:10.1080/15548627.2017.1293766.

9. Harry GJ. Microglia during development and aging. Pharmacol Ther. 2013;139(3):313-26. doi:10.1016/j.pharmthera.2013.04.013.

10. Hickman S, Izzy S, Sen P, Morsett L, Khoury E, J. Microglia in neurodegeneration. Nat Neurosci. 2018;21(10):1359-69. doi:10.1038/s41593-018-0242-x.

11. Nayak D, Roth TL, McGavern DB. Microglia development and function. Annu Rev Immunol. 2014;32:367-402. doi:10.1146/annurev-immunol-032713-120240.

12. Rupprecht R, Papadopoulos V, Rammes G, Baghai TC, Fan J, Akula N,.. . Schumacher M. Translocator protein (18 kDa) (TSPO) as a therapeutic target for neurological and psychiatric disorders. Nat Rev Drug Discov. 2010;9(12):971-88. doi:10.1038/nrd3295.

13. Liu GJ, Middleton RJ, Hatty CR, Kam WW, Chan R, Pham T,.. . Banati RB. The 18 kDa translocator protein, microglia and neuroinflammation. Brain Pathol. 2014;24(6):631-53. doi:10.1111/bpa.12196.

14. Wolf A, Herb M, Schramm M, Langmann T. The TSPO-NOX1 axis controls phagocyte-triggered pathological angiogenesis in the eye. Nat Commun. 2020;11(1):2709. doi:10.1038/s41467-02016400-8.

15. Ye C, Lin L, Zhang P, Chen Y, Huang J, Lin X. The protective effect of PK11195 on D-galactoseinduced amnestic mild cognitive impairment in rats. Ann Transl Med. 2020;8(18):1190. doi:10.21037/atm-20-6157.

16. Guilarte TR. TSPO in diverse CNS pathologies and psychiatric disease: A critical review and a way forward. Pharmacol Ther. 2019;194:44-58. doi:10.1016/j.pharmthera.2018.09.003.

17. Meyer JH, Cervenka S, Kim MJ, KreisI WC, Henter ID, Innis RB. Neuroinflammation in psychiatric disorders: PET imaging and promising new targets. Lancet Psychiatry. 2020;7(12):1064-74. doi:10.1016/s2215-0366(20)30255-8.

18. Gatliff J, Campanella M. TSPO: kaleidoscopic 18-kDa amid biochemical pharmacology, control and targeting of mitochondria. Biochem J. 2016;473(2):107-21. doi:10.1042/bj20150899.

19. Gatliff J, East D, Crosby J, Abeti R, Harvey R, Craigen W,... Campanella M. TSPO interacts with VDAC1 and triggers a ROS-mediated inhibition of mitochondrial quality control. Autophagy. 2014;10(12):2279-96. doi:10.4161/15548627.2014.991665.

20. Scaini G, Barichello T, Fries GR, Kennon EA, Andrews T, Nix BR,... Quevedo J. TSPO upregulation in bipolar disorder and concomitant downregulation of mitophagic proteins and NLRP3 inflammasome 
activation. Neuropsychopharmacology. 2019;44(7):1291-9. doi:10.1038/s41386-018-0293-4.

21. Feng H, Liu Y, Zhang R, Liang Y, Sun L, Lan N, Ma B. TSPO Ligands PK11195 and Midazolam Reduce NLRP3 Inflammasome Activation and Proinflammatory Cytokine Release in BV-2 Cells. Front Cell Neurosci. 2020;14:544431. doi:10.3389/fncel.2020.544431.

22. Papadopoulos $\mathrm{V}$, Lecanu L. Translocator protein ( $18 \mathrm{kDa}$ ) TSPO: an emerging therapeutic target in neurotrauma. Exp Neurol. 2009;219(1):53-7. doi:10.1016/j.expneurol.2009.04.016.

23. Lan N, Liu Y, Juan Z, Zhang R, Ma B, Xie K, Sun L, Feng H, Sun M, Liu J(2021). The TSPO-specific Ligand PK11195 Protects Against LPS-Induced Cognitive Dysfunction by Inhibiting Cellular Autophagy. Front Pharmacol.11, 615543. doi: 10.3389/fphar.2020.615543.

24. Wang Y, Liu N, Lu B. Mechanisms and roles of mitophagy in neurodegenerative diseases. CNS Neurosci Ther. 2019;25(7):859-75. doi:10.1111/cns.13140.

25. Plaza-Zabala A, Sierra-Torre V, Sierra A. (2017). Autophagy and Microglia: Novel Partners in Neurodegeneration and Aging. Int J Mol Sci, 18(3). doi:10.3390/ijms18030598.

26. Ghavami S, Shojaei S, Yeganeh B, Ande SR, Jangamreddy JR, Mehrpour M,.. . Łos MJ. Autophagy and apoptosis dysfunction in neurodegenerative disorders. Prog Neurobiol. 2014;112:24-49. doi:10.1016/j.pneurobio.2013.10.004.

27. Ising C, Venegas C, Zhang S, Scheiblich H, Schmidt SV, Vieira-Saecker A,.. . Heneka MT. NLRP3 inflammasome activation drives tau pathology. Nature. 2019;575(7784):669-73. doi:10.1038/s41586-019-1769-z.

28. Mangan MSJ, Olhava EJ, Roush WR, Seidel HM, Glick GD, Latz E. Targeting the NLRP3 inflammasome in inflammatory diseases. Nat Rev Drug Discov. 2018;17(8):588-606. doi:10.1038/nrd.2018.97.

29. Zhou R, Yazdi AS, Menu P, Tschopp J. A role for mitochondria in NLRP3 inflammasome activation. Nature. 2011;469(7329):221-5. doi:10.1038/nature09663.

30. Fan W, Mai L, Zhu X, Huang F, He H. The Role of Microglia in Perioperative Neurocognitive Disorders. Front Cell Neurosci. 2020;14:261. doi:10.3389/fncel.2020.00261.

31. Gong Z, Pan J, Shen Q, Li M, Peng Y. Mitochondrial dysfunction induces NLRP3 inflammasome activation during cerebral ischemia/reperfusion injury. J Neuroinflammation. 2018;15(1):242. doi:10.1186/s12974-018-1282-6.

32. Jian M, Kwan JS, Bunting M, Ng RC, Chan KH. Adiponectin suppresses amyloid- $\beta$ oligomer (ABO)induced inflammatory response of microglia via AdipoR1-AMPK-NF-KB signaling pathway. J Neuroinflammation. 2019;16(1):110. doi:10.1186/s12974-019-1492-6.

33. Yin Z, Han Z, Hu T, Zhang S, Ge X, Huang S,... Lei P. Neuron-derived exosomes with high miR-21-5p expression promoted polarization of M1 microglia in culture. Brain Behav Immun. 2020;83:270-82. doi:10.1016/j.bbi.2019.11.004.

34. Zujovic V, Taupin V. Use of cocultured cell systems to elucidate chemokine-dependent neuronal/microglial interactions: control of microglial activation. Methods. 2003;29(4):345-50. doi:10.1016/s1046-2023(02)00358-4. 
35. Zhou $F$, Zhang $C$, Guan $Y, \ldots$.Wang $X$. Screening the expression characteristics of several miRNAs in G93A-SOD1 transgenic mouse: altered expression of miRNA-124 is associated with astrocyte differentiation by targeting Sox2 and Sox9. J Neurochem. 2018;145(1):51-67.

doi:10.1111/jnc.14229.

\section{Figures}

a
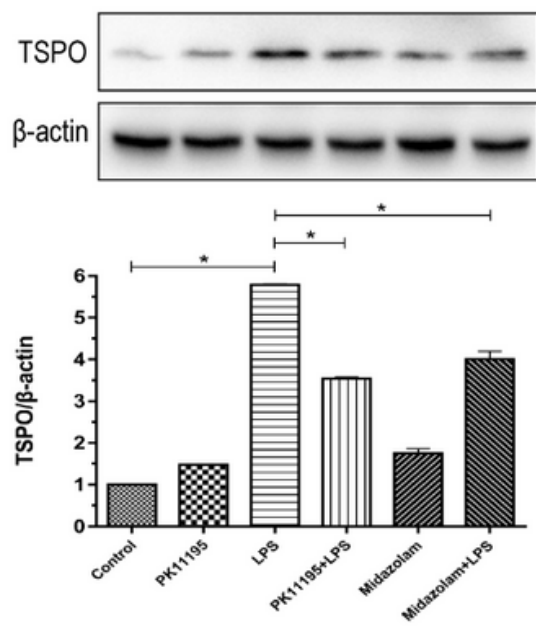

C

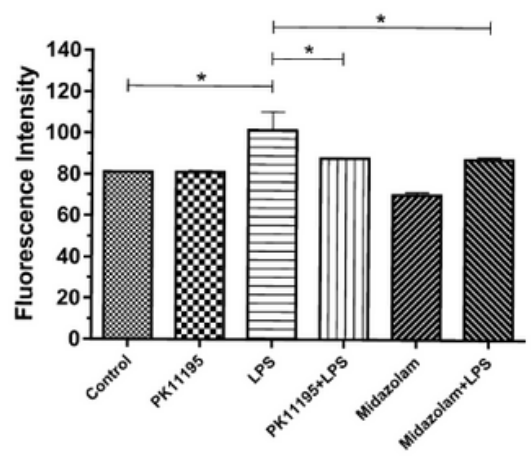

$f$ b

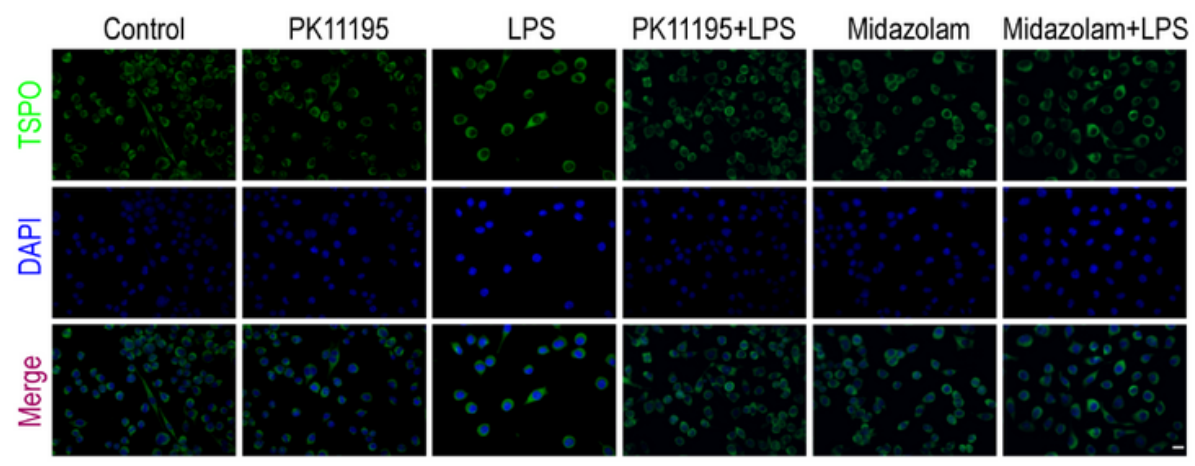

d

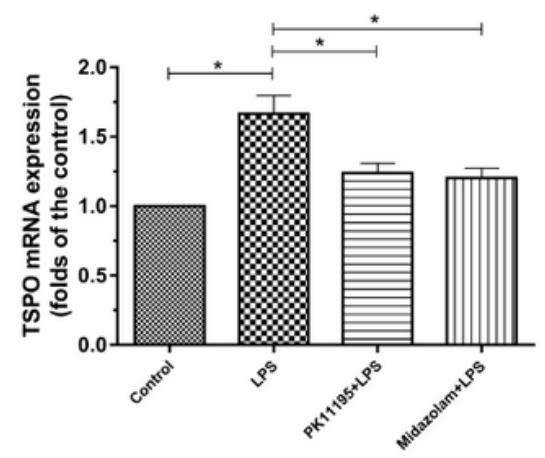

e
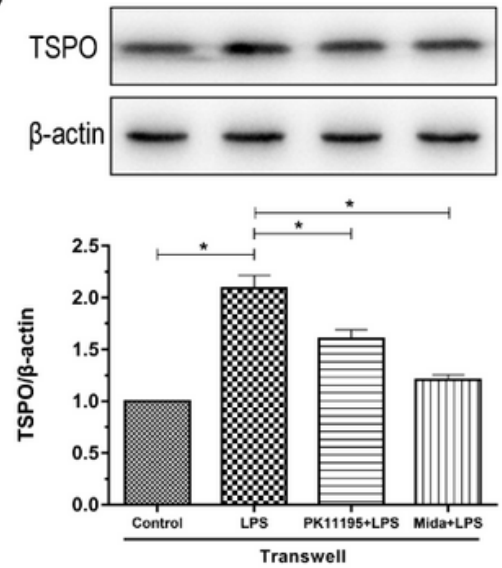

h
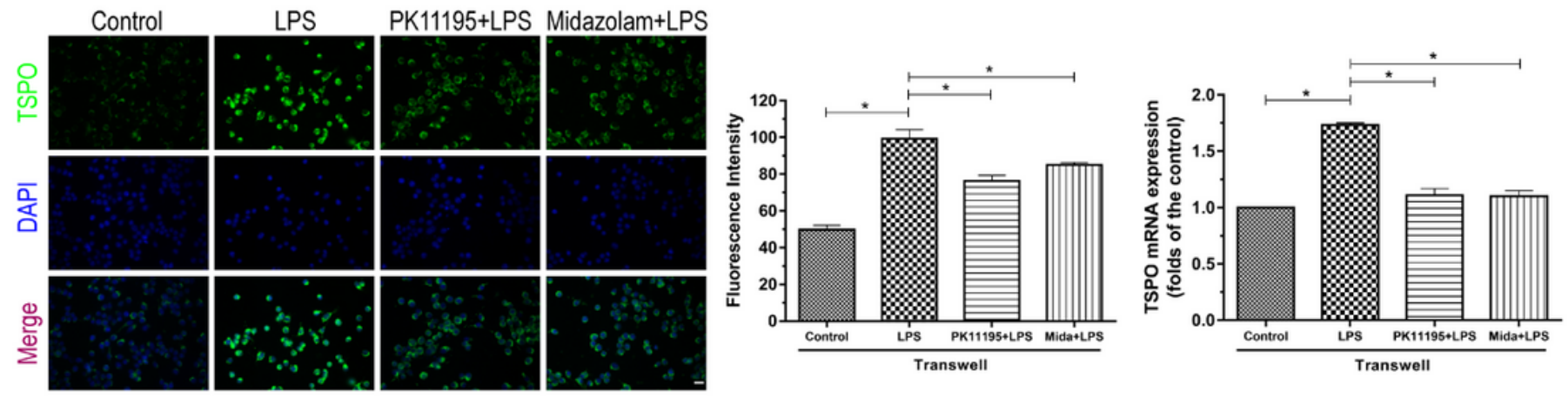

Figure 1 
TSPO ligands attenuated LPS-induced high TSPO expression in BV-2 microglia cells. The expression of TSPO were quantified by Western blot, Immunofluorescence and qRT-PCR. (a-d) The expression of TSPO in BV-2 microglia cells (e-h) The expression of TSPO in BV-2 microglia cell $\neg$ s in BV-2-NSC34 Transwell co-culture system. Scale bar, $20 \mu \mathrm{m} . * \mathrm{P}<0.05$

a

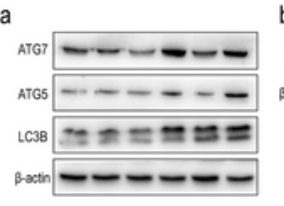

d

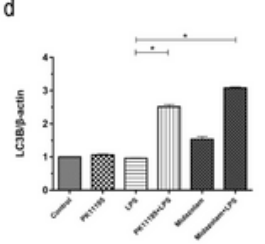

g

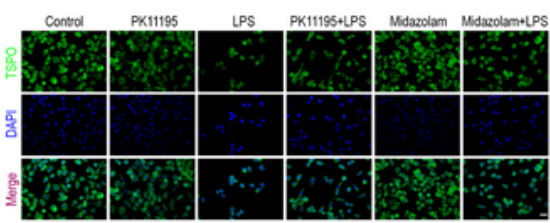

e
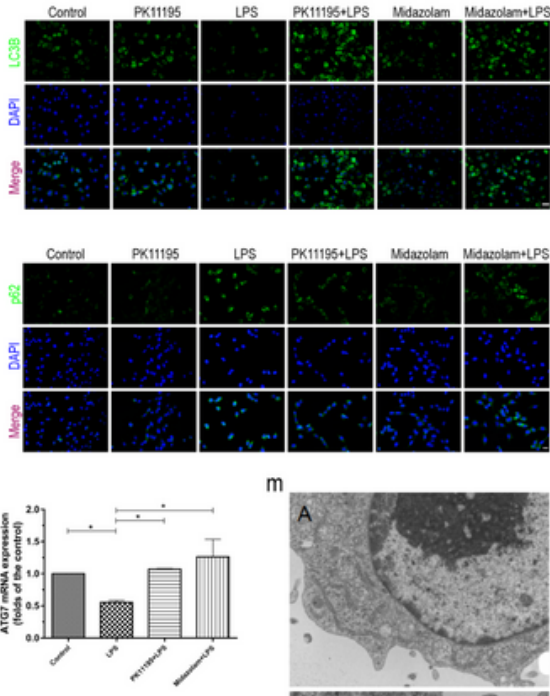

k
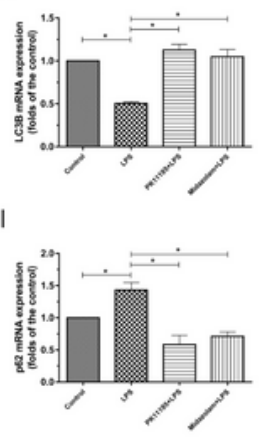

C

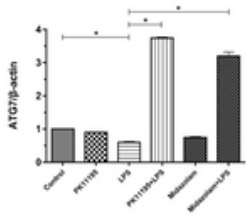

f
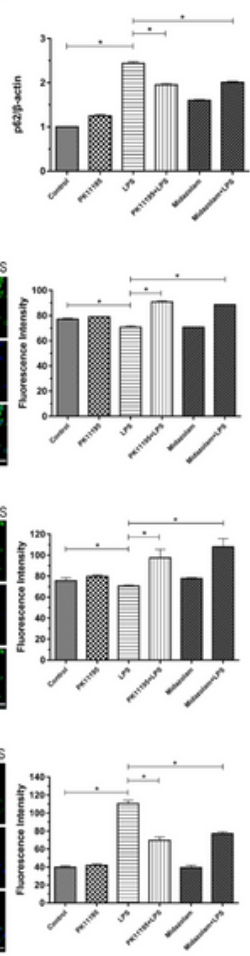

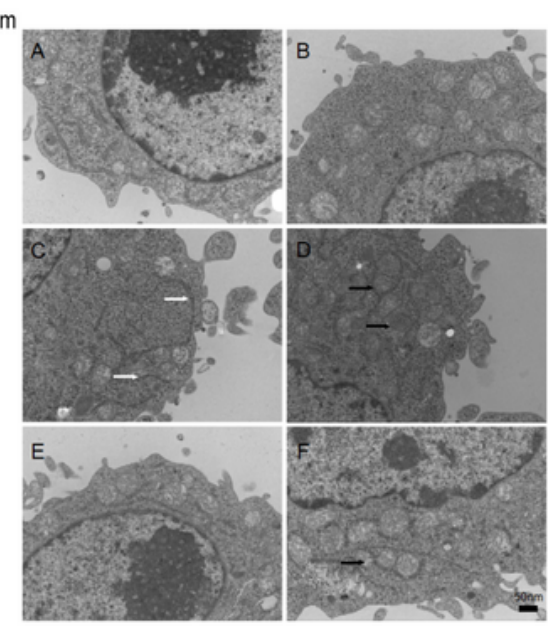

Figure 2 
TSPO ligands reversed the inhibition of mitophagy in LPS-activated BV-2 microglia cells. (a-f) Representative images of ATG7, ATG5, LC3B, p62 and $\beta$-actin expression in BV-2 microglia cells were detected by Western blot. (g-i) Representative images showing ATG7, LC3B and p62 expression with immunofluorescence in BV-2 microglia cells. Scale bar, $20 \mu \mathrm{m}$. $\neg$ ᄀר(j-I) Comparison of ATG7, LC3B and p62 expression in BV-2 microglia cells by groups using qRT- PCR. $(\mathrm{m})$ TEM was employed to detect the ultrastructure of cells. Scale bar, $50 \mathrm{~nm}$ A: Control B: PK11195 C: LPS D: PK11195+LPS E: Midazolam F: Midazolam+LPS. White solid arrow: swollen mitochondria; Black solid arrow: autophagic vacuoles. $* \mathrm{P}<$ 0.05 
a

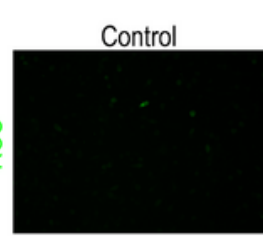

b

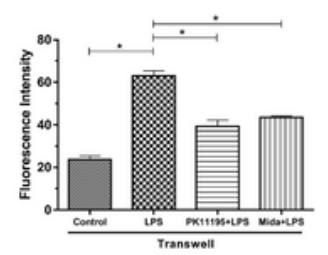

LPS

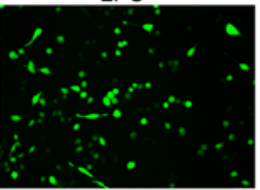

c

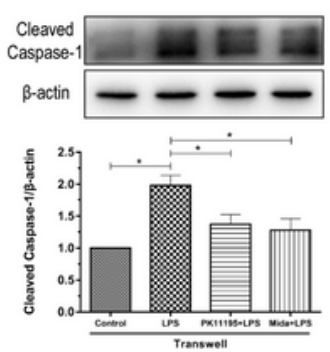

Midazolam+LPS
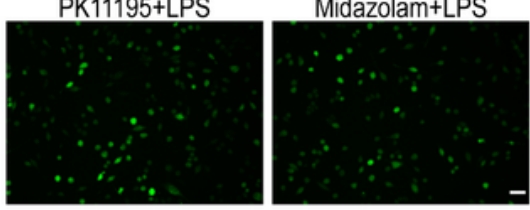

d

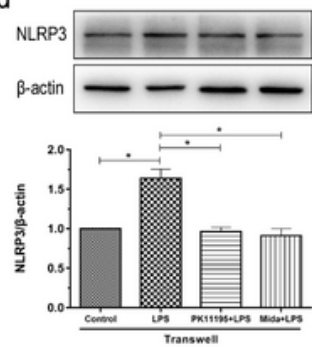

e
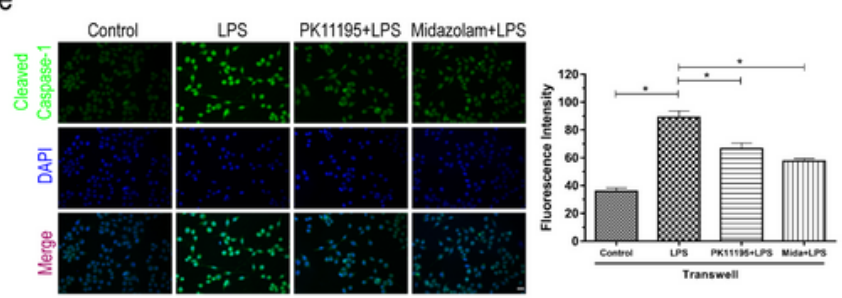

f
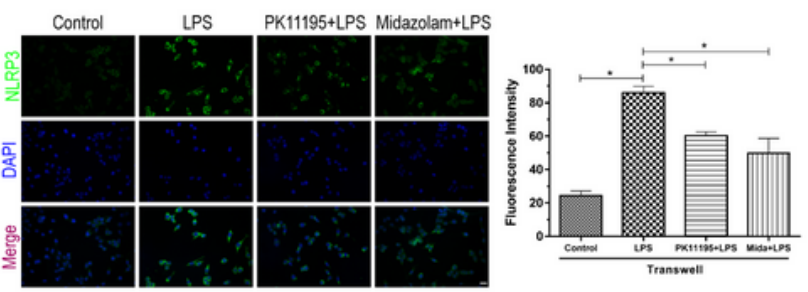

g

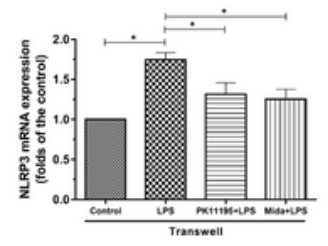

h

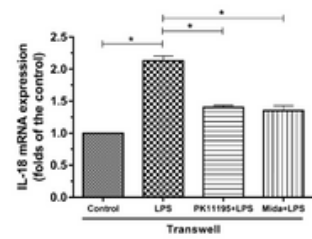

j
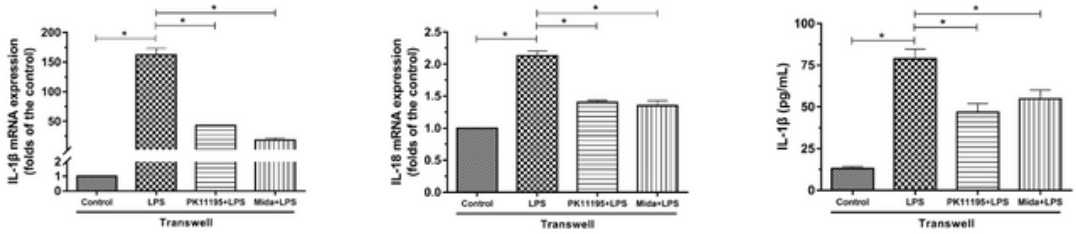

k

$$
\text { I }
$$

m
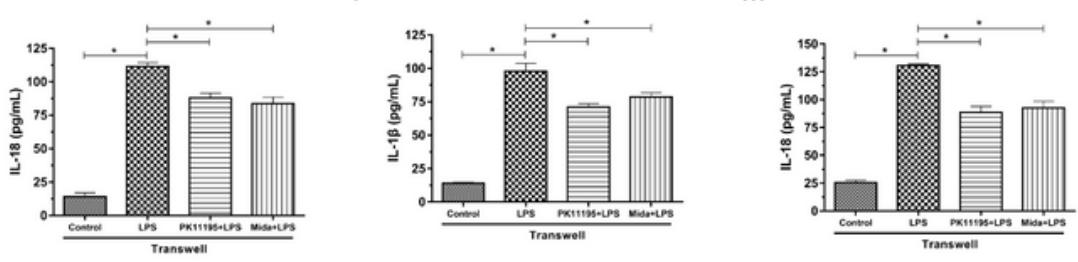

\section{Figure 3}

TSPO ligands inhibited neuroinflammatory reactions in LPS-activated BV-2 microglia cells in microglianeurons Transwell co-culture system. (a-b) Representative images showing the ROS production with DCFH-DA in BV-2 microglia cells in BV-2-NSC34 Transwell co-culture system. Scale bar, $20 \mu \mathrm{m}$. (c-d) Cleaved caspase-1 and NLRP3 expression in BV-2 microglia cells based on Western blot analysis. (e-f) Representative images showing Cleaved caspase- 1 and NLRP3 expression with immunofluorescence in 
BV-2 microglia cells. Scale bar, $20 \mu \mathrm{m}$. (g-i) The mRNA expression of NLRP3, IL-1 $\beta$ and IL-18 were determined by qRT-PCR in BV-2 microglia. ( $j-k$ ) The content of IL-1 $\beta$ and IL-18 in BV-2-NSC34 Transwell co-culture system was determined by ELISA. (I-m) The content of IL-1 $\beta$ and IL-18 in BV-2-HT-22 Transwell co-culture system was determined by ELISA. $* \mathrm{P}<0.05$

a

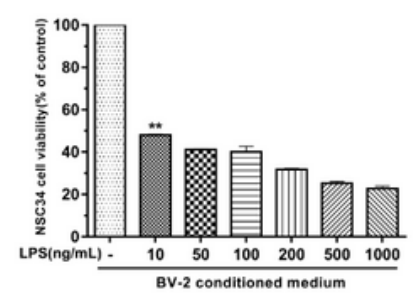

d

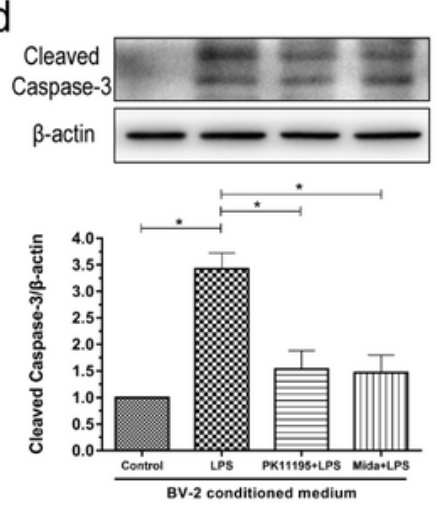

b

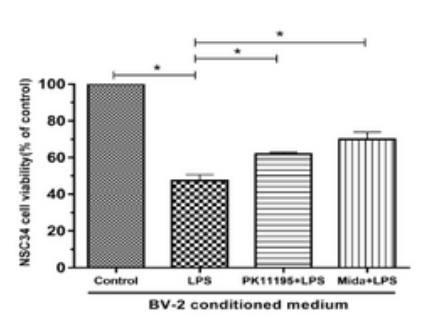

C
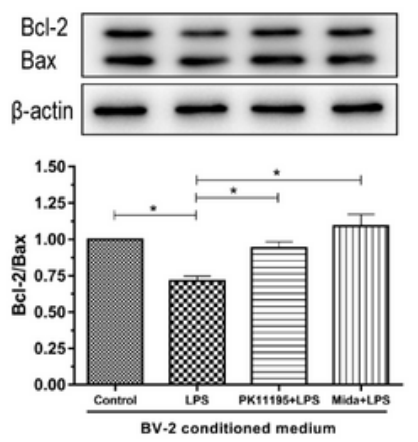

f
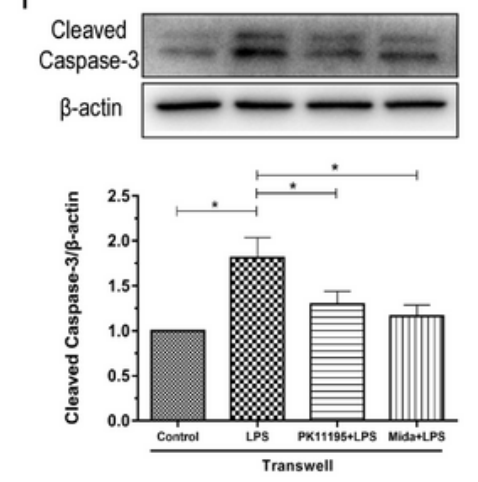

$f$

g

e
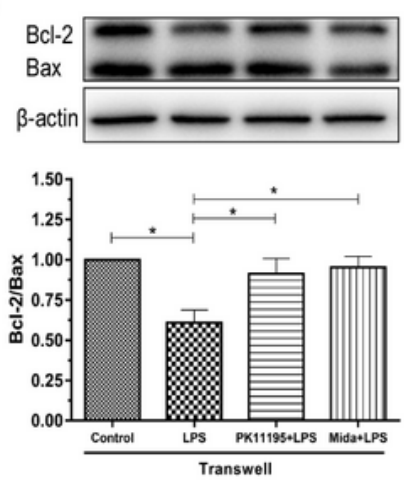
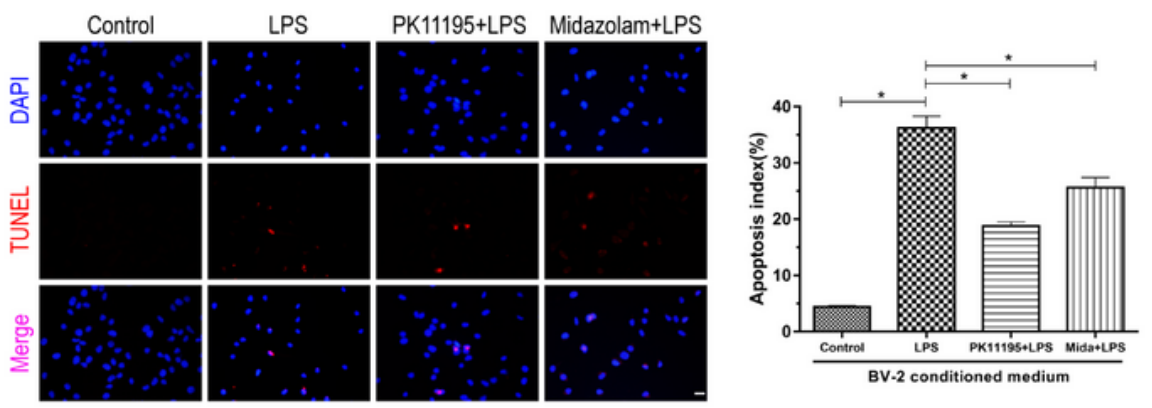

h
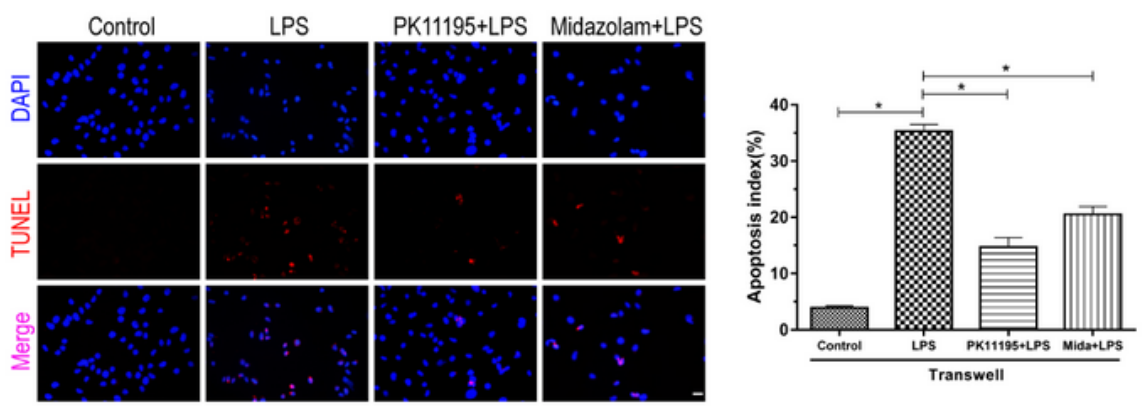

Figure 4 
Anti-inflammatory effects of PK11195 and Midazolam on LPS-activated BV-2 cells protected NSC34 neuronal cells from cytotoxicity and provided neuroprotective against the NSC34 neuronal cell apoptosis. (a-b) The NSC34 neuronal cell viability detected by CCK-8. (c-f) The relative expression of apoptosisrelated protein Bcl-2, Bax and Cleaved caspase-3 in NSC34 neuronal cells under CM (c, d) or Transwell (e, f) co-culture system was analyzed by Western blot. The NSC34 neuronal cells apoptosis was determined by TUNEL staining in CM (g) or Transwell (h) co-culture system. Scale bar, $20 \mu \mathrm{m} * \mathrm{P}<0.05,{ }^{* * \mathrm{P}<0.01}$

a

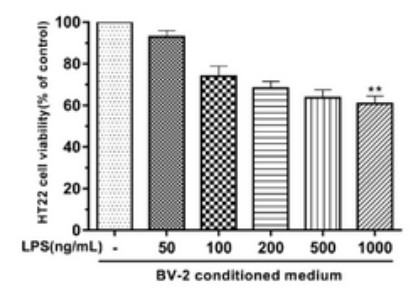

d

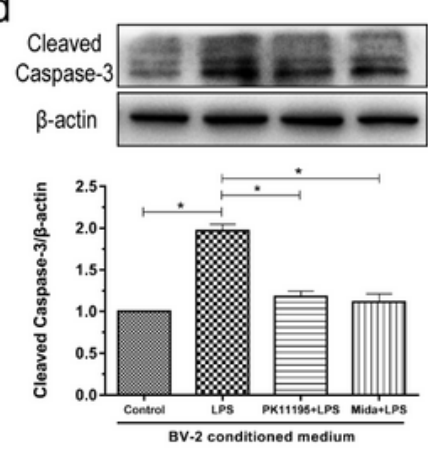

b

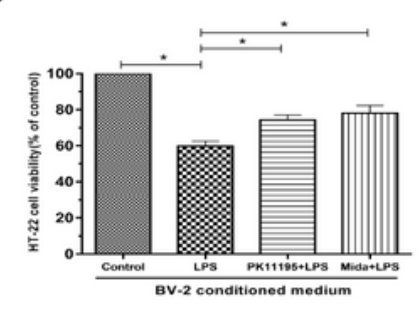

C

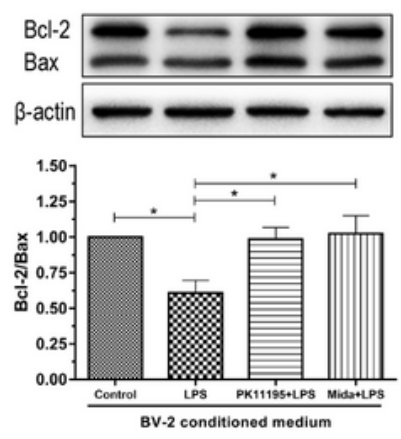

e
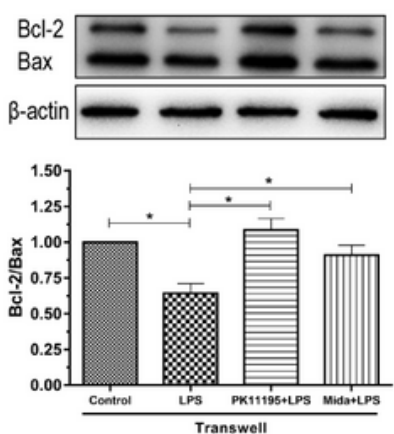
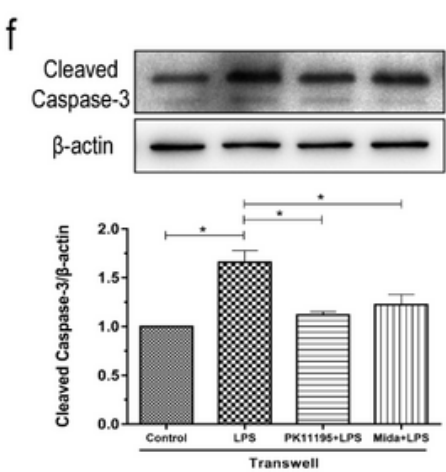

g
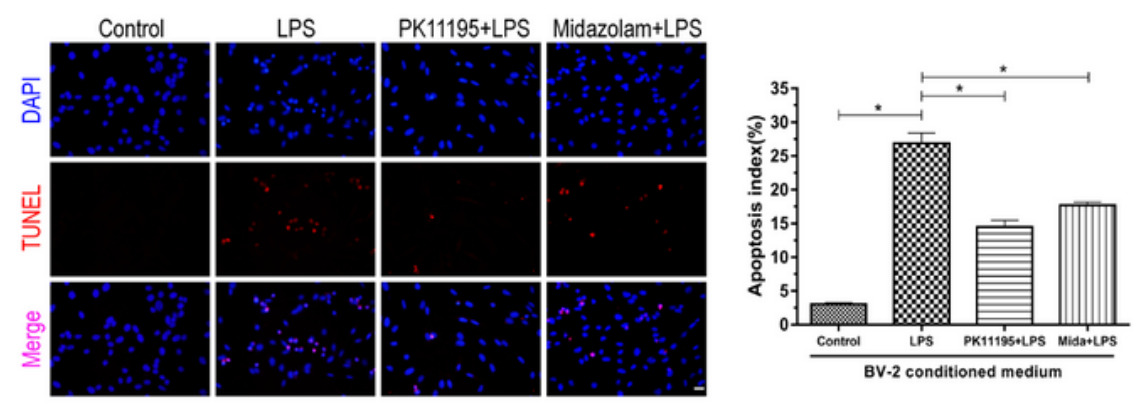

h
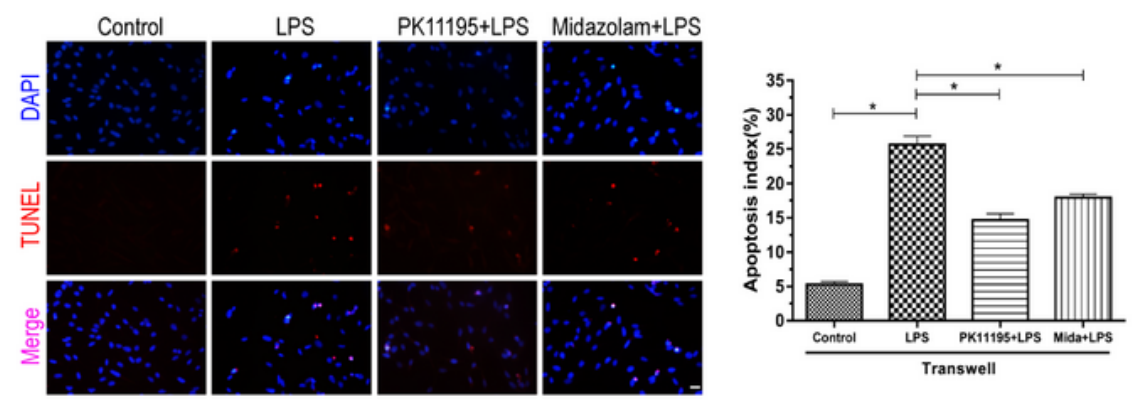

Figure 5 
Anti-inflammatory effects of PK11195 and Midazolam on LPS-activated BV-2 cells protected HT-22 neuronal cells from cytotoxicity and provided neuroprotective against the HT-22 neuronal cell apoptosis. (a-b) The HT-22 neuronal cell viability detected by CCK-8. (c-f) The relative expression of apoptosis-related protein Bcl-2, Bax and Cleaved caspase-3 in HT-22 neuronal cells under CM (c, d) or Transwell $(e, f)$ coculture system was analyzed by Western blot. The HT-22 neuronal cells apoptosis was determined by TUNEL staining in CM (g) or Transwell ( $h$ ) co-culture system. Scale bar, $20 \mu \mathrm{m} * \mathrm{P}<0.05,{ }^{*} \mathrm{P}<0.01$

\section{Supplementary Files}

This is a list of supplementary files associated with this preprint. Click to download.

- Supplementarymaterial.docx 